Sciendo

\title{
Uma Lógica da Indistinguibilidade
}

J.R. Arenhart, Universidade Federal da Fronteira Sul

D. Krause, Universidade Federal de Santa Catarina

Disputatio Vol. 4, No. 34

December 2012

DOI: $10.2478 /$ disp-2012-0020

ISSN: 0873-626X 


\title{
Uma Lógica da Indistinguibilidade
}

\author{
J.R. Arenhart \\ Universidade Federal da Fronteira Sul \\ D. Krause \\ Universidade Federal de Santa Catarina \\ BIBLID [0873-626X (2012) 34; pp. 555-573]
}

\section{Introdução}

Segundo uma concepção bastante em voga atualmente, a ontologia com a qual devemos nos comprometer deve de algum modo ser 'inferida' de nossas melhores teorias científicas, ou seja, dito de modo resumido, se desejamos saber o que há, devemos indagar às nossas melhores teorias. Esta posição, que é uma forma de naturalismo em ontologia, encontra algumas dificuldades no caso da mecânica quântica não-relativista. Segundo certas interpretações, o formalismo desta teoria é compatível com pelo menos dois tipos bastante distintos de ontologias: uma ontologia 'clássica' na qual as entidades tratadas por esta teoria são vistas como indivíduos em alguma acepção, ${ }^{1}$ ou alternativamente, também é possível defender que a teoria nos

\footnotetext{
${ }^{1}$ Porém, podendo ser indiscerníveis, alguma versão do Princípio da Identidade dos Indiscerníveis (PII), de Leibniz, seria supostamente violado por estas partículas, pelo menos segundo alguns autores (algumas referências sobre este tópico são apontadas em Muller \& Seevinck [2009], embora esses autores não concordem com a violação do PII pela mecânica quântica). O princípio nos garante que se objetos são numericamente distintos então deve existir alguma propriedade ou relação que os diferencie. Neste caso, se assumimos que as partículas quânticas são indivíduos, então o princípio de individualidade para elas não pode se basear em PII. Recentemente, no entanto, alguns autores tentaram estabelecer a validade de uma forma do PII neste contexto (ver Saunders [2006], Saunders e Muller [2008], Muller e Seevinck [2009]). No entanto, em nenhum desses trabalhos o conceito de "propriedade" é dado de forma rigorosa.
}

Disputatio, Vol. IV, No. 34, December 2012 
compromete com uma ontologia de não-indivíduos, ${ }^{2}$ objetos para os quais as leis da identidade, conforme formuladas na lógica clássica, não se aplicam irrestritamente (ver French e Krause [2006] cap. 4 e 6). O problema é que a contraparte matemática da teoria não nos dá indicações que permitam favorecer uma destas interpretações em detrimento da outra. Assim, aparentemente, no caso desta teoria, a disputa terá que ser travada no campo da argumentação filosófica.

Adotemos uma terminologia provisória e informal. Um indivíduo será aqui (redundantemente) entendido como uma entidade (por falta de um termo melhor) que possa pelo menos em princípio ser identificado de modo inequívoco em qualquer contexto, em especial, sendo diferente de todas as demais entidades que forem dele distintas. Por exemplo, se supusermos a teoria de conjuntos Zermelo-Fraenkel com o axioma da escolha (ZFC), então todo conjunto pode ser bem-ordenado, ${ }^{3}$ em particular o conjunto $\mathfrak{R}$ dos números reais é bem ordenado. O que acontece é que, como se sabe, não podemos exprimir essa boa ordem por uma fórmula da linguagem de ZFC. Em particular, como $(0,1) \subseteq \mathfrak{R}$, este subconjunto terá um menor elemento, que, no entanto, não pode ser expresso na linguagem (por exemplo, dando-lhe um nome). Porém, mesmo assim ele é um indivíduo na nossa acepção, sendo distinto de qualquer outro número real, como implica a lógica clássica, que subjaz à teoria usual dos números reais.

Por um não-indivíduo, entenderemos qualquer entidade que não obedece as condições acima. Não-indivíduos podem ser de diferentes 'espécies', e quando são indiscerníveis, ainda que possam ser co-

${ }^{2} \mathrm{O}$ termo não-indivíduo, apesar de não muito adequado, é o padrão nestas discussões. É utilizado para designar objetos que violam alguma forma do princípio de individuação, em particular, para objetos que violam as chamadas leis da identidade, e mais especificamente a propriedade reflexiva da identidade, ou seja, para qualquer objeto $\mathrm{x}, \mathrm{x}=\mathrm{x}$. Isso se deveria não ao fato de haver objetos que não são idênticos a eles mesmos, mas à circunstância de que a noção de identidade não poderia ser aplicada. Ver French \& Krause [2006] para uma discussão pormenorizada. Outro conceito importante e não muito claro nessas discussões é o de "lógica clássica”. Voltaremos a essa questão mais abaixo.

${ }^{3}$ Uma boa ordem sobre um dado conjunto é uma ordem parcial (reflexiva, anti-simétrica e transitiva) relativamente à qual todo subconjunto do conjunto dado tem menor elemento (um elemento do subconjunto que é menor-na ordem dada - que todos os outros elementos do subconjunto). 
ligidos em coleções da vários deles, por definição não poderão ser discernidos por quaisquer critérios que se imagine, nem mesmo em princípio. Um não-indivíduo pode aparecer em um contexto e, se de algum modo for substituído por algum outro de mesma espécie, o contexto não se altera. Obviamente, uma coleção de não-indivíduos não satisfaria o axioma da extensionalidade de uma teoria de conjuntos como ZFC. Claro que essas caracterizações são imprecisas, e suas definições rigorosas dependerão da linguagem e da lógica empregadas. Deste modo, não há uma única definição de indivíduo, e o mesmo se dá para a de não-indivíduo, mas isso não impede que busquemos tornar estes conceitos mais precisos utilizando algum aparato lógico.

Com efeito, um modo de concebermos um pouco mais rigorosamente o que são indivíduos, ainda que haja dificuldade de se caracterizar precisamente o que é a lógica clássica, ${ }^{4}$ seria sustentarmos que um indivíduo obedece as regras da teoria clássica da identidade, de primeira ordem ou de ordem superior (ou de alguma teoria de conjuntos calcada na lógica clássica). Assim, em especial, um não-indivíduo não obedece a auto-identidade, isto é, a lei fundamental $a=a$. Isso no entanto, segundo nossa caracterização, não implica que $a \neq a$, mas que a noção de identidade não se aplica aos não-indivíduos (o que está de acordo com a posição de Erwin Schrödinger, por exemplo, em Schrödinger [1952], pp.17-18; ver French \& Krause [2006]). Esta hipótese parece essencial para podermos caracterizar não-indivíduos. Com efeito, admitamos que eles podem estar relacionados por uma relação mais fraca de 'indiscernibilidade', que representaremos por ' $\equiv$ '. Assim, se supusermos, como parece razoável, que se $x \equiv y$ então eles são inter-substituíveis salva veritate, ou seja, vale algo como o axioma da substitutividade da igualdade da lógica de primeira ordem (a saber, algo como $x \equiv y \rightarrow(\alpha(x) \rightarrow \alpha(y))$--ver mais à frente), se assumirmos além disso que $\forall_{x}\left(x \equiv_{x}\right)$, restituiríamos os

${ }^{4}$ De modo geral, podemos dizer que por lógica clássica entendemos o cálculo usual de predicados de primeira ordem com ou sem igualdade (conforme axiomatizado, por exemplo, em Mendelson [1987]), ou alguns de seus subsistemas, como o cálculo proposicional clássico, ou mesmo sistemas de 'grande lógica' como as lógicas usuais de ordem superior ou as teorias usuais de conjuntos, como ZFC e mesmo a teoria de categorias como usualmente concebida. 
axiomas da igualdade da lógica elementar clássica, e então em nada estaríamos contribuindo efetivamente, mas apenas mudando a notação da igualdade para ' $\equiv$ '. Portanto, tendo-se em vista a discussão sobre a ontologia associada à MQ não-relativista, resulta que uma das dificuldades que surgem é que a lógica clássica, que é a lógica subjacente à mecânica quântica não-relativista, não parece compatível com uma ontologia de não-indivíduos, entendidos no sentido acima, pois a identidade sempre faz sentido para todas as entidades tratadas pela lógica clássica. Assim, se desejamos sustentar que uma ontologia de não-indivíduos é plausível, pelo menos no caso desta teoria em particular, parece razoável buscarmos por sistemas de lógica que nos permitam tratar de objetos para os quais a identidade e diferença não façam sentido.

Este tipo de investigação é importante filosoficamente, como atestam muitos autores, que sustentam que esta ontologia, a de não-indivíduos, é a mais natural para uma possível interpretação da mecânica quântica, principalmente se levarmos em conta determinadas interpretações de seus aspectos experimentais (ver a discussão histórica em French e Krause [2006] cap. 3). Isto torna imperiosa uma busca por mais rigor nos termos nos quais se expressam os conceitos de tal ontologia. Não entraremos aqui nas discussões sobre qual ontologia é mais adequada neste caso, nos restringindo aos problemas dos fundamentos lógicos da ontologia de não-indivíduos, que caracterizaremos abaixo.

Neste trabalho, apresentaremos uma linguagem formal de primeira ordem cujo objetivo principal é permitir o tratamento rigoroso de objetos para os quais a identidade e a diferença não se apliquem, ou seja, linguagens que possam tratar de não-indivíduos nesta acepção do termo. Este tipo de lógica é conhecido na literatura como não-reflexiva ou para-reflexiva (por exemplo, da Costa e Krause [1994], [1997] da Costa e Bueno [2009]). Vamos também considerar alguns problemas que surgem quando desejamos, ao mesmo tempo em que adotamos uma ontologia de não-indivíduos fazendo uso das linguagens por nós propostas, utilizar alguma das teorias de conjuntos clássicas $^{5}$ como metalinguagem para se estabelecer a semântica destas

${ }^{5}$ Grosso modo, estamos considerando como clássica uma teoria de conjuntos cuja lógica subjacente é a lógica clássica. 
linguagens. Aqui, estamos supondo que a ontologia com a qual nos compromete uma teoria é determinada pelas entidades que devem existir no domínio de quantificação para que as sentenças da teoria sejam verdadeiras, no sentido tarskiano. Como veremos, se o domínio de quantificação dado por uma estrutura na qual interpretamos uma teoria formulada utilizando-se o sistema de lógica aqui proposto for um conjunto no sentido clássico, então as entidades com a qual trata a teoria serão indivíduos, violando, de certo modo, a motivação para se utilizar a lógica proposta neste trabalho.

A linguagem que apresentaremos é uma pequena modificação de uma proposta feita primeiramente por Newton C. A. da Costa em seu livro Ensaio sobre os Fundamentos da Lógica, (da Costa [2008] pp. 138-141), e constitui-se basicamente de uma linguagem bissortida de primeira ordem com certas restrições que comentaremos adiante, dando origem a um sistema que foi por ele batizado de lógica de Schrödinger. O objetivo que tinha em mente ao apresentar esta lógica era mostrar que o princípio de identidade, conforme formalizado por certa formulação ${ }^{6}$, pode ser derrogado, ou seja, pode-se conceber um sistema de lógica, a lógica de Schrödinger, no qual a identidade ou diferença não se aplique a todas as entidades com as quais se pretende tratar. Este sistema de lógica era também motivado pelas dificuldades em se tratar da identidade e diferença quando falamos de partículas elementares, e deveria refletir certas intuições de E. Schrödinger quem, como dito acima, ao falar sobre partículas elementares da física quântica, insistia em que a questão sobre sua identidade ou diferença, em certos contextos, não faria sentido. Ou seja, (apesar de nem Schrödinger nem da Costa utilizarem esta terminologia), estes objetos são certo tipo de não-indivíduos: "Está além da dúvida que a questão da 'igualdade', da identidade [no que concerne as partículas elementares], real e verdadeiramente não tem sentido.” (Schrödinger [1952] p. 18).

\footnotetext{
${ }^{6}$ da Costa utilizava a propriedade reflexiva da identidade da lógica clássica de primeira ordem, ou seja, $\forall \mathrm{x}(\mathrm{x}=\mathrm{x})$, como uma forma de representar o princípio da identidade.
} 


\section{Uma lógica para a indistinguibilidade}

Adotaremos em nossa exposição a idéia original de da Costa para restringir a identidade de modo que faça sentido apenas para alguns objetos do domínio do discurso. Para fazer com que a identidade no sentido usual não se aplique a certas entidades, da Costa empregou uma linguagem bissortida, com duas espécies de termos individuais, e uma mudança na definição de fórmulas. Uma das espécies de termos, que podemos supor que seja a primeira, denotaria objetos microscópicos, e a outra, a segunda, denotaria as entidades macroscópicas. A restrição feita na definição de fórmula é a de impedir que o símbolo de identidade seja uma fórmula quando ladeado por pelo menos um termo de primeira espécie. Os axiomas da lógica clássica, observadas as diferenças de termos, completavam a apresentação. Em nosso caso, para formularmos uma lógica da indistinguibilidade, acrescentaremos ainda a esta linguagem uma relação binária de indistinguibilidade com postulados adequados para os objetos de primeira espécie. Os objetos de segunda espécie também poderão relacionar-se pela relação de indistinguibilidade, mas neste caso esta relação colapsará na identidade para estes objetos.

Para vermos como esta idéia funciona, apresentaremos agora uma linguagem de primeira ordem para uma lógica da Indistinguibilidade, que chamaremos abreviadamente L. Nosso sistema é baseado na lógica de Schrödinger, proposta por da Costa (da Costa [2008]) e generalizado para linguagens de ordem superior, como em da Costa e Krause [1994], [1997], e no sistema de lógica da Indiscernibilidade apresentado por Krause [2007] cap. 3. Utilizaremos os seguintes símbolos primitivos:

(a) Conectivos: $\rightarrow$ (implicação) e $\neg$ (negação);

(b) Quantificador universal: $\forall$ (para todo);

(c) Pontuação: ), (, , (parênteses e vírgula);

(d) Uma coleção enumerável de variáveis individuais de primeira espécie $\mathrm{x}_{1}, \mathrm{x}_{2}, \ldots, \mathrm{x}_{\mathrm{n}}, \ldots$, e uma coleção qualquer de constantes individuais de primeira espécie $\mathrm{a}_{1}, \mathrm{a}_{2}, \ldots, \mathrm{a}_{\mathrm{n}}, \ldots$; claro que este modo de falar pode ser tornado adequadamente preciso e independente de noções como 'enumerável'. Por exemplo, para variáveis de primeira espécie poderíamos usar os 
símbolos $x$ e $\mid$, de forma que as variáveis de primeira espécie seriam expressões (sequencias finitas de símbolos) da forma $x, x|, x| \mid$, etc. O mesmo pode ser dito das demais situações similares aqui apresentadas.

(e) Uma coleção enumerável de variáveis individuais de segunda espécie $\mathrm{X}_{1}, \mathrm{X}_{2}, \ldots, \mathrm{X}_{\mathrm{n}}, \ldots$, e uma coleção qualquer de constantes individuais de segunda espécie $A_{1}, A_{2}, \ldots, A_{n}, \ldots$;

(f) O símbolo de predicado binário ' $=$ ' para a identidade e o símbolo de predicado binário ‘ $\equiv$ ' para indistinguibilidade;

(g) Para cada número natural $\mathrm{n}>0$, uma coleção eventualmente vazia de símbolos de predicados de peso $\mathrm{n}$.

Os outros conectivos, disjunção, conjunção e bi-implicação podem ser definidos da maneira usual, assim como o quantificador existencial. Um termo é uma variável ou uma constante individual. Os termos podem ser divididos, de modo evidente, em termos de primeira e segunda espécie. Por brevidade, usaremos as letras $t_{1}, t_{2}, t_{3}$, etc., como meta-variáveis para termos de qualquer das duas espécies, e $\mathrm{x}, \mathrm{y}$ e $\mathrm{z}$ sem índices como meta-variáveis para variáveis de qualquer das duas espécies. Outra convenção que passaremos a utilizar será denotar por 'm-termos' os termos de primeira espécie e 'M-termos' os termos de segunda espécie.

Intuitivamente, os m-termos representarão as entidades básicas da microfísica, tal como descritas por alguma versão da mecânica quântica não-relativística, e os M-termos representarão os objetos macroscópicos. O objetivo, como comentamos acima, é fazer com que a identidade se aplique apenas a $\mathrm{M}$-termos, e não a m-termos, pois para estes não faria sentido falar em identidade ou em diversidade, e que a indistinguibilidade se aplique a todos os objetos, desde que sejam da mesma espécie. Formalmente, isso se obtém ao se impedir, na definição de fórmula, que $t_{i}=t_{i}$ seja bem formada caso $t_{i}$ ou $\mathrm{t}_{\mathrm{j}}$ sejam m-termos, e ao impormos que $\mathrm{t}_{\mathrm{i}}$ e $\mathrm{t}_{\mathrm{j}}$ sejam da mesma espécie para que $t_{i} \equiv t_{j}$ seja bem formada. Com exceção destas restrições, a definição de fórmula também segue a usual, e como se pode notar a partir dos postulados que daremos, a lógica clássica se aplica de maneira usual aos M-termos. Mais especificamente, temos: 
Definição [Fórmulas atômicas] Se $t$. e $t$. são termos da mesma espécie, então $t \equiv t$ é fórmula atômica. Se $t_{i}$ e $t_{j}$ são termos de segunda espécie, então $t_{i}=t_{j}$ é fórmula atômica. Se $\mathrm{P}$ é um símbolo de predicados de peso $n$, outro que a identidade ou a indistinguibilidade, e $t_{1}$...t são $\mathrm{n}$ termos, então $\mathrm{Pt}_{1} \ldots \mathrm{t}_{\mathrm{n}}$ é fórmula atômica.

Definição [Fórmulas] As fórmulas de L são: (i) As fórmulas atômicas; (ii) Se $\alpha$ é fórmula, $\neg \alpha$ é fórmula; (iii) Se $\alpha$ e $\beta$ são fórmulas, então $\alpha \rightarrow \beta$ é fórmula; (iv) Se $\alpha$ é uma fórmula e x é uma variável, então $\forall \mathrm{x} \alpha$ é uma fórmula; (v) Apenas são fórmulas as expressões dadas pelas cláusulas anteriores.

É importante enfatizar: $\mathrm{t}_{\mathrm{i}}=\mathrm{t}_{\mathrm{j}}$ será fórmula apenas se ti e tj forem ambos termos de segunda espécie. Nossa definição de fórmula proíbe que expressões como, por exemplo, $\mathrm{x}_{1}=\mathrm{X}_{1}$, ou $\mathrm{A}_{2}=\mathrm{x}_{1}$ ou ainda $\mathrm{A}_{1}=\mathrm{a}_{2}$ sejam bem formadas. No entanto, como dissemos anteriormente, a relação de indistinguibilidade vai se manter entre os dois tipos de objetos. Teremos que $t_{i} \equiv t_{j}$ sempre será uma fórmula bem formada, mas devemos observar a restrição de que $t_{i}$ e $t_{j}$ sejam de mesma espécie, ou seja, a definição de fórmula proíbe que, por exemplo, $\mathrm{x}_{1} \equiv \mathrm{X}_{1}$, $\mathrm{A}_{2} \equiv \mathrm{x}_{1}$ e $\mathrm{A}_{1} \equiv \mathrm{a}_{2}$ sejam fórmulas.

O seguinte conjunto de postulados pode ser utilizado para L (outra formulação de uma lógica de primeira ordem com uma relação de indistinguibilidade pode ser encontrada nos postulados para a lógica da Indiscernibilidade, proposta em Krause [2007] cap. 3):

1. $\alpha \rightarrow(\beta \rightarrow \alpha)$

2. $\alpha \rightarrow(\beta \rightarrow \gamma) \rightarrow((\alpha \rightarrow \beta) \rightarrow(\alpha \rightarrow \gamma))$

3. $((\neg \alpha \rightarrow \neg \beta) \rightarrow((\neg \alpha \rightarrow \beta) \rightarrow \alpha))$

4. $\alpha \rightarrow \beta, \alpha / \beta(\mathrm{MP})$

5. $\forall \mathrm{x} \alpha(\mathrm{x}) \rightarrow \alpha(\mathrm{t})$, com $\mathrm{x}$ e t da mesma espécie, e $\mathrm{t}$ livre para $\mathrm{x}$ em $\alpha(\mathrm{x})$.

6. $\beta \rightarrow \alpha(x) / \beta \rightarrow \forall x \alpha(x)$, onde $x$ não ocorre livre em $\beta$.

7. $\mathrm{t}=\mathrm{v}_{\mathrm{v}} \rightarrow(\alpha(\mathrm{t}) \rightarrow \alpha(\mathrm{v}))$, com t e v termos de segunda espécie, além das restrições usuais.

8. $\forall \mathrm{x}(\mathrm{x} \equiv \mathrm{x})$.

9. $\forall \mathrm{x} \forall \mathrm{y}(\mathrm{x} \equiv \mathrm{y} \rightarrow \mathrm{y} \equiv \mathrm{x})$.

10. $\forall \mathrm{x} \forall \mathrm{y} \forall \mathrm{z}(\mathrm{x} \equiv \mathrm{y} \wedge \mathrm{y} \equiv \mathrm{z} \rightarrow \mathrm{x} \equiv \mathrm{z})$.

11. $\forall \mathrm{X} \forall \mathrm{Y}(\mathrm{X} \equiv \mathrm{Y} \rightarrow \mathrm{X}=\mathrm{Y})$. 
É importante perceber que os postulados que envolvem a relação de identidade estão formulados para termos de segunda espécie, principalmente o postulado 11, que intuitivamente significa que se dois macro-objetos são indiscerníveis, então eles são idênticos. Em breve veremos que a recíproca é teorema de $\mathrm{L}$.

Conceitos sintáticos como os de demonstração, dedução a partir de um conjunto de fórmulas, teorema, ocorrências livres e ligadas de variáveis entre outros também são definidos da maneira usual. O Teorema da Dedução também pode ser demonstrado da maneira usual.

Temos agora o prometido teorema de que, para M-objetos, a igualdade implica a indistinguibilidade.

Teorema. $\mathrm{X}=\mathrm{Y} \rightarrow \mathrm{X} \equiv \mathrm{Y}$

\section{Demonstração:}

1. $\mathrm{X}=\mathrm{Y}$ (hipótese)

2. $\mathrm{X}=\mathrm{Y} \rightarrow(\mathrm{X} \equiv \mathrm{X} \rightarrow \mathrm{X} \equiv \mathrm{Y})$ (postulado 7)

3. $(\mathrm{X} \equiv \mathrm{X} \rightarrow \mathrm{X} \equiv \mathrm{Y})(1,2$ Modus Ponens $)$

4. $\forall \mathrm{X}(\mathrm{X} \equiv \mathrm{X})$ (postulado 8)

5. $\forall \mathrm{X}(\mathrm{X} \equiv \mathrm{X}) \rightarrow \mathrm{X} \equiv \mathrm{X}$ (postulado 5)

6. $\mathrm{X} \equiv \mathrm{X}(4,5$ Modus Ponens)

7. $\mathrm{X} \equiv \mathrm{Y}(3,6$ Modus Ponens)

8. $\mathrm{X}=\mathrm{Y} \rightarrow \mathrm{X} \equiv \mathrm{Y}$ (1-7 Teorema da Dedução)

Assim, com este teorema e o postulado 11, temos que para M-objetos, $\mathrm{X}=\mathrm{Y} \leftrightarrow \mathrm{X} \equiv \mathrm{Y}$, conforme prometemos anteriormente. No entanto, para $\mathrm{m}$-objetos, este bicondicional não pode ser demonstrado. Ainda, neste caso, a relação de indistinguibilidade é caracterizada apenas como uma relação de equivalência, sem valer necessariamente o esquema da substituição dado pelo postulado 7. Isto a caracteriza sintaticamente como uma relação mais fraca do que a identidade. No entanto, como ainda não especificamos como devemos interpretar estes símbolos, nada impede, certamente, que ao se fazer uma semântica para L se interprete a relação de indistinguibilidade também como a relação de identidade, que satisfaz os axiomas de L dados para o símbolo de indistinguibilidade.

Também temos como teorema de L a reflexividade da identidade, que não precisa ser postulada. Com isto, a relação de identidade possui em $\mathrm{L}$ as duas propriedades que são usualmente utilizadas como 
axiomas para a relação de identidade na lógica clássica de primeira ordem: reflexividade e substituição. Com estas propriedades, como se sabe, é possível demonstrar, por exemplo, que a relação de identidade é simétrica e transitiva.

Teorema. $\mathrm{X}=\mathrm{X}$

\section{Demonstração:}

1. $\forall \mathrm{X}(\mathrm{X} \equiv \mathrm{X})$ (postulado 8$)$

2. $\forall \mathrm{X}(\mathrm{X} \equiv \mathrm{X}) \rightarrow(\mathrm{X} \equiv \mathrm{X})$ (postulado 5$)$

3. $\mathrm{X} \equiv \mathrm{X}(1,2$ Modus Ponens $)$

4. $\forall \mathrm{X}(\mathrm{X} \equiv \mathrm{X} \rightarrow \mathrm{X}=\mathrm{X})$ (postulado 11)

5. $\forall \mathrm{X}(\mathrm{X} \equiv \mathrm{X} \rightarrow \mathrm{X}=\mathrm{X}) \rightarrow(\mathrm{X} \equiv \mathrm{X} \rightarrow \mathrm{X}=\mathrm{X})$ (postulado 5)

6. $(\mathrm{X} \equiv \mathrm{X} \rightarrow \mathrm{X}=\mathrm{X})(4,5$ Modus Ponens $)$

7. $\mathrm{X}=\mathrm{X}(3,6$ Modus Ponens)

Não faremos a demonstração da simetria e transitividade da identidade para $\mathrm{M}$-objetos aqui, pois o procedimento é o mesmo que na lógica clássica. Uma demonstração alternativa para estes fatos pode ser fornecida utilizando-se a equivalência para $\mathrm{M}$-objetos entre identidade e indistinguibilidade e os postulados 8 e 9.

É interessante notar também que a lógica clássica de primeira ordem está de certo modo 'contida' na lógica L. Isto ocorre pelo fato de que, intuitivamente, os postulados de $\mathrm{L}$, quando restritos aos $\mathrm{M}$ -termos, podem ser tomados como um conjunto de postulados para a lógica clássica. Falando mais rigorosamente, é possível se estabelecer uma tradução da lógica clássica em L, mostrando que os postulados da lógica clássica, quando traduzidos em $\mathrm{L}$, são teoremas de $\mathrm{L}$.

Sem dificuldade, podemos estender nossa lógica a uma lógica de ordem superior (teoria simples de tipos) e a uma teoria de conjuntos, que será em muito semelhante à teoria de quase-conjuntos $Q$ (French e Krause [2006], cap.7).

\section{Semântica clássica para L e seus problemas}

Ao apresentar seu sistema de lógica, da Costa discutia uma interpretação pretendida, que deveria ser erigida de modo que as intuições básicas que serviram de base para se formular esta lógica fossem pre- 
servadas. As variáveis individuais de segunda espécie percorreriam um conjunto no sentido usual, e as constantes individuais de segunda espécie nomeariam elementos deste conjunto. Por outro lado, as variáveis de primeira espécie deveriam percorrer uma coleção de não-indivíduos, e as constantes de primeira espécie deveriam nomear tais elementos. Os símbolos de relação de peso n, como usual, denotariam coleções de n-uplas de elementos destas coleções, e o símbolo de relação de indistinguibilidade, em nosso caso, deve denotar uma relação que simule a indistinguibilidade.

Este procedimento, no entanto, se conduzido da maneira usual, utilizando uma teoria de conjuntos ao estilo de ZFC como metalinguagem, suscita vários problemas filosóficos, caso queiramos preservar as intuições que deram origem à lógica de Schrödinger e a lógica da Indistinguibilidade, pois parece inviabilizar nosso comprometimento com uma ontologia de não-indivíduos relativamente aos objetos denotados pelos termos de primeira espécie. Isto ocorre, entre outros motivos, porque, como veremos com mais detalhe abaixo, nas teorias de conjuntos usuais, nas quais usualmente fundamentamos a semântica para linguagens formais como as que estamos discutindo, a identidade sempre faz sentido para todos os elementos do conjunto, e assim acabamos re-introduzindo a identidade para estes objetos através da metalinguagem.

Para discutirmos com mais rigor estes problemas, apresentamos a partir de agora um esboço de uma semântica clássica para L, onde o termo 'clássica' refere-se ao fato de que esta semântica é formulada na teoria de conjuntos ZFC. Como este modo de proceder é bastante conhecido, não seremos rigorosos e faremos apenas o suficiente para que possamos apresentar adiante, com mais detalhes, os problemas ocasionados por esta semântica quando desejamos tratar alguns elementos do domínio como não-indivíduos. Devemos enfatizar que as dificuldades aqui apresentadas não são uma exclusividade da lógica L e da lógica de Schrödinger, mas sim uma dificuldade que qualquer sistema de lógica para-reflexiva deve enfrentar. Em geral, como estes sistemas violam alguma forma do princípio de identidade, a semântica formal feita para eles deveria ser tal que os objetos do domínio da estrutura na qual interpretamos a linguagem preservassem esta característica. No entanto, como veremos no caso particular que estamos tratando, dificuldades surgem quando desejamos estabelecer 
uma semântica para estes sistemas utilizando uma teoria de conjuntos clássica (ver também as discussões em da Costa e Bueno [2009]).

Procedendo da maneira conhecida, e seguindo as sugestões de da Costa ([2008] pp. 140, 141), queremos interpretar os símbolos não-lógicos da linguagem de $\mathrm{L}$ em uma estrutura $\mathbf{e}=<\mathrm{D}$, I $>$, onde:

1. D é um conjunto não-vazio que vai fazer o papel de domínio da estrutura. No presente caso, impomos ainda que $\mathrm{D}=$ $\mathrm{D}_{1} \cup \mathrm{D}_{2}$, com $\mathrm{D}_{1} \cap \mathrm{D}_{2}=\varnothing$. Assumimos que as variáveis de primeira espécie tomam valores em $\mathrm{D}_{1}$, e as de segunda em $\mathrm{D}_{2}$.

2. Quanto a I, ela será a função denotação, e atribui às relações de identidade e indistinguibilidade e aos símbolos não-lógicos da linguagem elementos de $\mathrm{D}$ da seguinte forma:

2.1. Aos símbolos de predicados de peso $\mathrm{n}$ da linguagem de $\mathrm{L}$, outros que a identidade e a indistinguibilidade, a função I associa da maneira usual um subconjunto de $\mathrm{D}^{\mathrm{n}}$.

2.2. Temos que: $I\left(a_{i}\right) \in D_{1}$, ou seja, às constantes de primeira espécie associam-se elementos de $\mathrm{D}_{1}$, e $\mathrm{I}\left(\mathrm{A}_{\mathrm{i}}\right) \in \mathrm{D}_{2}$, ou seja, às constantes de segunda espécie associam-se elementos de $\mathrm{D}_{2}$.

2.3. Ao símbolo de identidade associamos o conjunto $\{<\mathrm{x}, \mathrm{y}\rangle$ : $\mathrm{x}, \mathrm{y} \in \mathrm{D}_{2}$ e $\left.\mathrm{x}=\mathrm{y}\right\}$.

2.4. Ao símbolo de indistinguibilidade $\equiv$ atribuímos uma relação $\mathrm{R}$ em $\mathrm{D}$ tal que $\mathrm{R}$ é relação de equivalência.

Com a semântica acima esboçada, é possível obter resultados como a correção e completude de L com relação a esta semântica, da maneira usual, com as convenientes adaptações para a linguagem bissortida (Mendelson [1987] Cap. 2). No entanto, do ponto de vista filosófico, há uma série de problemas com esta semântica, problemas esses que surgem quando interpretamos os termos de primeira espécie como denotando entidades quânticas, tendo-se em vista que queremos sustentar, seguindo nossa leitura de Schrödinger e de outros autores, que elas são certo tipo de não-indivíduos. Alguns destes problemas já foram apontados por da Costa (da Costa [2008] pp. 140, 141), e outros ainda podem ser encontrados em Krause [2002].

Passamos agora a apresentar alguns destes problemas. Com eles, desejamos sugerir que é relevante buscar-se uma semântica mais ade- 
quada para L do ponto de vista de suas motivações, ou seja, uma semântica na qual estes problemas possam ser superados. Uma das alternativas propostas na literatura é que se empregue como metalinguagem a Teoria de Quase-Conjuntos, uma teoria que permite formar coleções de objetos indistinguíveis mas não idênticos, mas não entraremos nestes detalhes neste trabalho (ver French e Krause [2006], cap. 7 e 8).

Do ponto de vista das motivações que deram origem à lógica da indistinguibilidade, o primeiro problema com essa semântica clássica começa com a escolha do domínio: nosso conjunto $\mathrm{D}_{1}$ não poderia ser um conjunto no sentido comum das teorias de conjuntos usuais, em particular, não poderia ser um conjunto de ZF, a teoria de conjuntos que se utiliza usualmente como metalinguagem. Isto já havia sido apontado por da Costa ([2008], p. 140), e se deve ao fato de que essas teorias estão comprometidas com uma noção cantoriana de conjunto, no sentido de que conjuntos são coleções de objetos distintos uns dos outros, o que pressupõe a validade irrestrita da teoria da identidade para estes objetos e para seus elementos. Isto pode ser visto também como resultando do fato de que a lógica subjacente à teoria de conjuntos ZFC e todas as outras teorias de conjuntos clássicas é a lógica clássica, na qual a identidade se aplica sem restrições a todos os objetos, impedindo que para alguns deles, que deveriam representar os não-indivíduos, a identidade ou diferença não se aplique.

Ainda, é importante perceber que de acordo com o princípio de extensionalidade, dois conjuntos são idênticos se e somente se tiverem os mesmos elementos, o que depende, como se vê, de um conceito sensato de identidade. Além disso, os axiomas da teoria de conjuntos utilizada implicam que sempre é possível formar o conjunto unitário de um elemento dado, que será diferente do conjunto unitário de qualquer outro elemento (distinto do primeiro) por extensionalidade. Ou seja, um 'conjunto clássico' é um conjunto de indivíduos (no sentido por nós definido anteriormente), distinguíveis uns dos outros, para os quais a identidade sempre faz sentido. Para expressar este ponto com mais rigor, pode-se dizer que $<\mathrm{V}, \in>$, o 'modelo' pretendido para ZF, se visto como uma estrutura matemática, ${ }^{7}$

${ }^{7}$ Este ponto, no entanto, é sutil. Não podemos elaborar um modelo de ZF na própria ZF (suposta consistente), como atesta o segundo teorema de incomple- 
é uma estrutura rígida, mas não entraremos em detalhes aqui (ver Krause e Coelho [2005]).

Caso adotemos em nosso domínio o conjunto $\mathrm{D}_{1}$ como um 'conjunto clássico', estaremos claramente reintroduzindo a identidade para estes elementos via metamatemática, ainda que queiramos impedir esse fato em nossa linguagem objeto. Desta forma, como salientou da Costa, estaremos abandonando a motivação inicial segundo a qual a identidade não deveria fazer sentido para certas entidades (que seriam os elementos de $\mathrm{D}_{1}$ ). É importante enfatizar isto: uma das motivações para se propor L era possibilitar que se tratasse sensatamente com não-indivíduos, e se esta motivação não for observada na metalinguagem, aparentemente, não se terá restringido nada com as mudanças sintáticas da linguagem de L (para discussões ainda mais gerais sobre este tópico, ver da Costa, Bueno e Béziau [1995]). ${ }^{8}$

Outro aspecto no qual a maneira usual de fazer semântica entra em conflito com as motivações de L diz respeito à interpretação das constantes. Se quisermos que a identidade não tenha sentido para os elementos de $\mathrm{D}_{1}$, não é possível que a função interpretação atribua a cada constante de primeira espécie um único e bem determinado elemento de $\mathrm{D}_{1}$. Isto ocorre porque se os elementos de $\mathrm{D}_{1}$ forem imaginados como denotando os quanta, de acordo com a interpretação que estamos supondo, não faz sentido nomeá-los desta maneira, pois se forem indistinguíveis, a princípio não podemos identificá-los nem distingui-los. Explicando um pouco mais este ponto, o problema é que podemos dar um 'nome' para uma partícula, por exemplo, cha-

tude de Gödel. Os modelos de ZF, caso existam, devem ser buscados em teorias mais fortes. Para certos conjuntos de axiomas, no entanto, podemos encontrar 'modelos internos' (no sentido de Gödel), mas não discutiremos este ponto aqui.

${ }^{8}$ Este tipo de situação ocorre freqüentemente quando se propõe um sistema de lógica que viole alguma das chamadas leis da lógica usual. Em geral, a metalinguagem utilizada para se fazer a semântica para estes sistemas pode ser considerada como sendo ZF, que pressupõe a validade da lei que se pretende derrogar, e acaba por nos comprometer com esta lei na metalinguagem. Por exemplo, na lógica intuicionista, desejamos entre outras coisas, que a lei do terceiro excluído não tenha validade geral, mas, se fizermos semântica para esta lógica em ZF, estaremos nos comprometendo, na metalinguagem, com a validade irrestrita desta lei, o que não é intuicionisticamente aceitável. Assim, semânticas distintas, que sejam aceitáveis de um ponto de vista intuicionista devem ser buscadas. 
mando Eddy (nome bastante usado na literatura, bem como Priscilla e Astrid) a um elétron aprisionado em um aparato laboratorial. No entanto, esse 'nome' não resiste a uma eventual permutação de partículas, por exemplo, desfazendo e refazendo o experimento. ${ }^{9}$ Neste caso a questão sobre se o Eddy preso no segundo experimento é ou não o mesmo que estava aprisionado no aparato quando fizemos o experimento pela primeira vez simplesmente não faz sentido, pois neste contexto os nomes não podem operar como designadores rígidos. (Isso também é salientado em Dalla Chiara1985; ver French e Krause op.cit., p.225).

Insistindo um pouco neste ponto, o problema não é simplesmente que se fizermos a suposição de que seja possível atribuir nomes no sentido usual para os quanta, haveria um problema em saber qual, dentre vários elementos indistinguíveis, é o portador de determinado nome, pois isso denotaria unicamente uma limitação epistemológica. O problema, no caso dos não-indivíduos, é ainda mais sério do que este; é que para eles, segundo a interpretação que estamos adotando, esta questão deixa de ser significativa, pois quando seguimos a interpretação acima apontada de Schrödinger, não faz sentido perguntar nem mesmo qual dentre várias entidades indistinguíveis recebeu o nome, e então a questão torna-se um problema de indeterminação ontológica, e insistir na rotulação através de nomes seria desistir da suposição de que estas entidades são realmente não-indivíduos segundo a nossa caracterização.

O problema com os nomes, mencionado acima, gera ainda outro grande inconveniente quando se pretende dar seqüência às definições anteriores. Usualmente, dois caminhos se apresentam. Por um lado, podemos definir a verdade para sentenças através do uso da linguagem diagrama relativamente ao domínio de interpretação, ampliando-se a linguagem ao acrescentar novos nomes, um para cada elemento do domínio, e então fornecer as cláusulas usuais por indução ou, por outro lado, podemos fazer esta definição através da noção de satisfatibilidade de uma fórmula por seqüências infinitas de elementos do domínio. Ambas alternativas são problemáticas do ponto de vista de nossos objetivos.

Com relação ao primeiro caminho apresentado, se não podemos

${ }^{9}$ Para mais discussões sobre este ponto, ver Krause [2006]. 
nomear os elementos de $\mathrm{D}_{1}$, como já apontamos acima, também não é possível ampliar a linguagem de $\mathrm{L}$ com constantes individuais que sejam cada uma delas um nome para um elemento determinado do domínio, pois em particular, pelo mesmo argumento acima, não faz sentido dar um nome para cada elemento de $\mathrm{D}_{1}$. Deste modo, caso decidamos nos manter fielmente de acordo com as motivações de L, caso adotemos uma postura 'schrödingeriana' com respeito às partículas, que por motivos de argumentação supomos que pertencem a $\mathrm{D}_{1}$, então nos parece não ser possível formar a linguagem diagrama de L.

A situação descrita no parágrafo anterior não melhora caso se opte pelo segundo caminho apresentado acima, qual seja, definir a verdade para sentenças através da relação de satisfatibilidade de uma fórmula por seqüências de objetos do domínio. Na verdade, seria possível, seguindo-se este caminho, ter uma atitude ainda mais radical, optando-se por abandonar completamente qualquer referência a nomes, eliminando-se da linguagem todas as constantes de primeira espécie (e também de segunda), tendo-se em vista alguns dos argumentos acima. No entanto, como dissemos, caso resolva-se prosseguir desta maneira para definir a relação de satisfatibilidade, utilizando-se seqüências infinitas de objetos do domínio, tal como pode ser feito no caso usual (ver Mendelson [1987] cap. 2), os problemas com relação às motivações subjacentes a $\mathrm{L}$ não desaparecem.

Neste caso, o problema é que não se podem formar as seqüências de objetos sem especificar quais são os objetos, sem rotulá-los, uma vez que devemos ter claro em qualquer seqüência, para fins da definição de satisfação, qual é o seu n-ésimo elemento para qualquer n, ou seja, é preciso identificá-lo rotulando-o, e novamente voltamos ao problema mencionado acima de que os não-indivíduos não são rotuláveis deste modo. Ainda, no mesmo sentido, como uma seqüência é uma função do conjunto dos naturais no conjunto domínio, é preciso que para cada n natural se atribua um único elemento de $\mathrm{D}$, em particular, de $\mathrm{D}_{1}$, e isto significa que para cada natural esteja bem especificado o elemento de $\mathrm{D}$ que lhe corresponde, dadas as características de uma função em ZF, e que tenhamos critérios, novamente, de identificação para os elementos de $\mathrm{D}_{1}$.

Um terceiro inconveniente com o qual nos deparamos diz respeito à atribuição de símbolos de predicados a subconjuntos de $\mathrm{D}$, 
ou seja, à especificação de extensões dos predicados da linguagem que se está interpretando. Novamente trata-se de conflito entre as motivações para a lógica L e a maneira usual de se fazer semântica. O problema é que não podemos dizer que certo predicado que verse sobre entidades quânticas determina um único conjunto com, digamos, n elementos. Na semântica usual todos os predicados são precisos ("sharp", para contrastá-los com predicados vagos), no sentido de que atribuímos a cada símbolo de predicados da linguagem um subconjunto de $\mathrm{D}^{\mathrm{n}}$ (a extensão do predicado), de modo que qualquer n-upla de elementos do domínio pertence à extensão de um predicado n-ário ou não pertence (dado que o princípio do terceiro excluído vale na semântica calcada na lógica usual). No entanto, “...na física quântica, existem certos predicados que são sharp no sentido de que os físicos sabem muito bem quais condições um indivíduo deve obedecer para ter a propriedade associada pelo predicado, mas [aparentemente] existem objetos vagos, que nos induzem a considerar um tipo de 'ignorância ontológica' neste caso. Isto mostra que a relação entre os predicados (que estão pelas intensões de certos conceitos) e as suas correspondentes extensões (o conjunto dos indivíduos que tem a propriedade atribuída pelo predicado) se torna distinta da semântica standard.” (Krause [2002] p. 78).

Assim, a situação da extensão de predicados, no caso da semântica que visa tratar de objetos da física quântica, é tal que não podemos determinar um conjunto bem definido de n-uplas para cada símbolo de predicados. Ainda temos, como no caso clássico que, dada uma n-upla de elementos do domínio, ela satisfaz ou não um predicado deste tipo. No entanto, para qualquer n-upla que pertence à extensão do predicado, outras $\mathrm{n}$-uplas, indistinguíveis da primeira mas que podem não pertencer à coleção que determina a extensão também satisfarão o predicado. A satisfação de um predicado por uma n-upla depende mais do tipo de objetos que compõe a n-upla do que do fato de eles pertencerem ou não à extensão do predicado, pois todas as n-uplas de um certo tipo satisfarão um predicado, caso alguma delas satisfaça, ou seja, se uma n-upla de elementos do domínio satisfaz o predicado, então, qualquer n-upla indistinguível dela também satisfará. 


\section{Conclusão}

Tendo em vista problemas como os mencionados acima, que se originam quando tentamos estabelecer uma semântica clássica para L de forma a manter as intuições que deram origem a esta lógica, segundo a qual os objetos com os quais trata a mecânica quântica não-relativista são certo tipo de não-indivíduos, aparentemente duas alternativas se sugerem, e que nos possibilitam superar estas dificuldades: abandonar o comprometimento com a ontologia de não-indivíduos e aceitar as dificuldades que surgem quando se adota esta posição relativamente à mecânica quântica, ou mudar a metalinguagem na qual se estabelece a semântica para linguagens como a de L. Newton da Costa sugeriu que, tendo em vista estas dificuldades, se criasse uma teoria de Quase-Conjuntos (da Costa [2008] p. 140), na qual fosse possível tratar de coleções de objetos indistinguíveis. Esta teoria pode ser vista em French e Krause [2006], cap. 7. Nela é possível erigir uma semântica para L que permite manter suas motivações metafísicas, mas a apresentação desta teoria e sua discussão estão fora do escopo deste trabalho. Este modo de proceder, apesar de nos permitir superar algumas das dificuldades apresentadas aqui e dar rigor aos termos nos quais uma ontologia de não-indivíduos pode ser formulada, ainda assim não encerra o debate filosófico sobre qual ontologia é a mais adequada para a Mecânica Quântica. No entanto, acreditamos que sistemas de lógica como o proposto, e outros, como a lógica de Schrödinger e a teoria de Quase-Conjuntos, contribuem para que a opção por uma ontologia de não-indivíduos se torne cada vez mais razoável, e para que formalismos que estejam mais de acordo com esta ontologia sejam erigidos para contribuir na discussão metafísica.

J.R. Arenhart Universidade Federal da Fronteira Sul

D. Krause Universidade Federal de Santa Catarina 


\section{Referências}

da Costa, N.C.A. 1979. Ensaio sobre os fundamentos da lógica, São Paulo, Hucitec/ EdUSP $3^{a}$. Ed. 2008.

da Costa, N.C.A. e Bueno, O. 2009. Lógicas não-reflexivas. In Revista Brasileira de Filosofia, ano 58, volume 232, 181-196.

da Costa, N.C.A. e Bueno, O., Béziau, J-Y. 1995. What is semantics? A brief note on a huge question. In Sorites - Electronic Quarterly of Analytical Philosophy 3, p. 43-47.

da Costa, N.C.A. e Krause, D. 1994. Schrödinger logics. In Studia Logica 53 (4), 1994, 533-550.

da Costa, N.C.A. e Krause, D. 1997. An Intensional Schrödinger Logic. In Notre Dame Journal of Formal Logic 38 (2), 1997, pp. 179-194.

Dalla Chiara, M.L. 1985. Names and descriptions in quantum logic. In Recent Developments in Quantum Logics, ed. by P. Mittelstaedt and E.W. Stachow. Manheim: Bibliografishes Institut, 189-202.

French, S. e Krause, D. 2006. Identity in Physics. A historical, philosophical and formal analysis. Oxford: Oxford University Press.

Krause, D. 2002. Why quasi-sets? In Boletim da Sociedade Paranaense de Matemática v. $201 / 2,2002,73-92$

Krause, D. 2006. Is Priscilla, the trapped positron, an individual? Quantum physics, the use of names, and individuation. A aparecer em Arbor (Spain).

Krause, D. 2007. La metafísica de los quanta. Manuscrito em preparo.

Krause, D., Coelho, A.M.N. 2005. Identity, Indiscernibility and Philosophical Claims. In Axiomathes 15 (2) pp. 191-210.

Mendelson, E. 1987. Introduction to Mathematical Logic. $3^{a}$ ed., Wadsworth \& Brooks.

Muller, F.A. e Saunders, S. 2008. Discerning Fermions. In The British Journal for the Philosophy of Science, 59(3) pp. 499-548.

Muller, F.A. e Seevinck, M.P. 2009. Discerning Elementary Particles. A aparecer em Philosophy of Science.

Saunders, S. 2006. Are quantum particles objects? In Analysis, 66.1, January, pp. 52-63.

Schrödinger, E. 1952. Science and Humanism. Cambridge: Cambridge Un. Press. 